\title{
Metric-Space Approach for Distinguishing Quantum Phase Transitions in Spin-Imbalanced Systems
}

\author{
T. de Picoli ${ }^{1} \cdot$ I. D $^{\prime}$ Amico $^{1,2}$ • V. V. França ${ }^{3}$ (I)
}

Received: 28 February 2018 / Published online: 26 July 2018

(C) Sociedade Brasileira de Física 2018

\begin{abstract}
Metric spaces are characterized by distances between pairs of elements. Systems that are physically similar are expected to present smaller distances (between their densities, wave functions, and potentials) than systems that present different physical behaviors. For this reason, metric spaces are good candidates for probing quantum phase transitions, since they could identify regimes of distinct phases. Here, we apply metric space analysis to explore the transitions between the several phases in spinimbalanced systems. In particular, we investigate the so-called FFLO (Fulde-Ferrel-Larkin-Ovchinnikov) phase, which is an intriguing phenomenon in which superconductivity and magnetism coexist in the same material. This is expected to appear for example in attractive fermionic systems with spin-imbalanced populations, due to the internal polarization produced by the imbalance. The transition between FFLO phase (superconducting phase) and the normal phase (non-superconducting) and their boundaries have been subject of discussion in recent years. We consider the Hubbard model in the attractive regime for which density matrix renormalization group calculations allow us to obtain the exact density function of the system. We then analyze the exact density distances as a function of the polarization. We find that our distances display signatures of the distinct quantum phases in spin-imbalanced fermionic systems: with respect to a central reference polarization, systems without FFLO present a very symmetric behavior, while systems with phase transitions are asymmetric.
\end{abstract}

Keywords Quantum phase transitions · FFLO superconductivity · Spin-imbalanced systems · Metric spaces

\section{Introduction}

Quantum phase transitions are characterized by sudden changes in the physical properties of the system driven by one of its parameters. The transition may be of first order, which has a discontinuity in the first derivative with respect to the parameter, or it may be smoother, as in secondorder transitions and crossovers [1]. Ideally, to investigate a quantum phase transition, one needs to obtain the order parameter, but this is not trivial in most of the cases. Therefore, it is quite common to use other properties of the system for example witnesses of quantum phase transitions,

$\triangle$ V. V. França

vvfranca@iq.unesp.br

1 São Carlos Institute of Physics, University of São Paulo, São Carlos, SP, 13560-970, Brazil

2 Department of Physics, University of York, York, YO10 5DD, UK

3 Institute of Chemistry, São Paulo State University, Araraquara, São Paulo, 14800-060, Brazil such as quantum correlations, entanglement measures, and Fisher information [2-8].

In this context, metric-spaces analysis appears as a powerful mathematical tool to investigate these transitions. In metric space, one can assign distances between wave functions, densities, and external potentials of two systems, which quantify the closeness between the systems. Recently, natural distances have been proposed for physical systems [9-11] and applied in several contexts [12-19].

In spin-imbalanced fermionic systems, several interesting phases might emerge across the imbalance strength. In the regime of attractive interactions, in particular, the system may present a conventional superfluid phase, as described by the Bardeen-Cooper-Schrieffer theory (BCS phase) [20], a normal magnetic (non-superfluid) phase, a fully polarized (FP) phase, a phase separation [21, 22], and a fascinating phenomenon denominated Fulde-FerrellLarkin-Ovchinnikov (FFLO) phase [23, 24]. The FFLO phase is characterized by the exotic coexistence of superconductivity and magnetism and expected to survive against the normal regime for small imbalances. Experimentally, there is only indirect evidences of the FFLO phase: in 
solid-state materials [25] and in one-dimensional Fermi gases [26].

From the theoretical point of view, regardless of the complexity of handling many-particle interactions and the harmonic confinement necessary to describe state-of-theart experiments, considerable understanding of the FFLO general properties has been achieved [4, 27-32]. However, the regime of polarizations at which the FFLO phase can be found is still under debate, as well as the nature of the transition to the other phases of the spin-imbalanced system.

Here, we apply a metric-space analysis to investigate the transitions between BCS, FFLO, normal, and FP phases in spin-imbalanced systems described by the onedimensional fermionic Hubbard model. We obtain the exact density functions of finite but large chains via density matrix renormalization group (DMRG) calculations and thus calculate density distances between systems with different polarizations. We find that the metric spaces are a suitable tool to detect quantum phase transitions. Our results suggest $(i)$ that the BCS-normal and the FFLO-normal phase transitions are of first order and (ii) that the normal-FP transition is a second-order phase transition or even a mere crossover.

\section{Theoretical and Computational Methods}

We consider the one-dimensional homogeneous Hubbard model [33]:

$H=-t \sum_{i, \sigma}\left(\hat{c}_{i, \sigma}^{\dagger} \hat{c}_{i+1, \sigma}+\hat{c}_{i+1, \sigma}^{\dagger} \hat{c}_{i, \sigma}\right)+U \sum_{i} \hat{n}_{i, \uparrow} \hat{n}_{i, \downarrow}$

where $t$ is the hopping parameter, $U$ is the intra-site interaction, and $\hat{c}_{i, \sigma}^{\dagger}$ and $\hat{c}_{i, \sigma}$ are creation and annhilation operators of fermionic particles at site $i$ with $z$-spin component $\sigma$, up $(+1 / 2)$ or down $(-1 / 2)$. Although it is one of the simplest model to describe itinerant and interacting particles in a chain, the Hubbard model describes very important phenomena [34] and has been proved to model properly nanostructures [35-40] and disordered systems [41].

We focus on finite chains, with size $L=80$, polarization (or imbalance) quantified by

$P=\frac{N_{\uparrow}-N_{\downarrow}}{N}$,

fixed number of particles $N=N_{\uparrow}+N_{\downarrow}$ and average particle density (filling factor) $n=N / L$. Here, the spatial inhomogeneity is only due to finite size effects and the density profile is obtained via DMRG techniques [42].

In all calculations, we will use attractive interactions $(U<0)$ in units of $t$ and set $t=1$. At $P=0$, the system is expected to be a conventional BCS superfluid, while at $P=1$, a fully polarized magnetic system. For intermediate polarizations, we should have the FFLO phase up to a certain critical value, $P_{C}$ [29], and a normal nonsuperfluid phase for $P>P_{C}$. The metric-space analysis will be performed here to distinguish between these several phases.

We thus consider the metric for densities as discussed in Ref. [9]:

$D\left(\rho_{1}, \rho_{2}\right)=\frac{1}{2 N} \int\left|\rho_{1}(\mathbf{r})-\rho_{2}(\mathbf{r})\right| d \mathbf{r}$,

where the scaling factor $2 N$ was added such that distances are within $[0,1]$ to facilitate straightforward comparisons. Here, $\rho_{1}$ and $\rho_{2}$ are the particle density functions of any two systems: the more dissimilar the systems, the greater the distance.

In order to identify the several phases that emerge by sweeping the polarization $P$, we explore the distance between densities corresponding to different $P$ 's. We use the central polarization value, $P=0.5$, to define the reference system, $\rho_{\text {ref }} \equiv \rho_{P=0.5}$ and thus quantify the density distance between this central reference and the system at $P$ :

$D(P)=\frac{1}{2 N} \int\left|\rho_{P}(\mathbf{r})-\rho_{r e f}(\mathbf{r})\right| d \mathbf{r}$.

The choice of the reference system is very important, as it defines which similarities and differences of the systems will be revealed by the distance measure. We have chosen it such that it does not correspond to any of the $P$ 's related to the transitions between distinct phases (neither $P=0$, $P=P_{C}$, nor $P=1$ ) and we have also avoided a reference system within the FFLO regime $\left(0<P<P_{C}\right.$, where $P_{C}^{\max }=1 / 3$ as shown in Ref. [29]). This particular choice: (i) avoids to emphasize characteristics of only one of the phase transitions, (ii) avoids possible strong fluctuations due to the inherent inhomogeneity of the FFLO phase, and (iii) allows us to explore a possible symmetry: in the absence of any phase transition, i.e., if the system presents only the normal phase within $0<P<1$, there should exist a symmetric behavior with respect to the central point $P=$ 0.5 , once the differences would be triggered exclusively by $P$.

\section{Results and Discussion}

We start by applying our density distance (4) in systems without FFLO phase. This may occur when the attractive interaction is too small (here, we use $U=-1$ ), such that the systems undergo directly a transition from the BCS superfluid to the normal phase. So, our systems without FFLO are characterized by three phases: BCS at $P=0$, 
normal non-superfluid phase for $0<P<1$, and fully polarized magnetic phase at $P=1$.

In the upper panel of Fig. 1, we present the density distance as a function of $P$ for systems without FFLO phase. The distance is essentially symmetric with respect to the central point for $0.1 \lesssim P \lesssim 0.9$, confirming the fact that there is no phase transition within $0<P<1$. In contrast though, there is a clear asymmetry between the BCS and the FP cases. This can be seen quantitatively in the upper panel of Fig. 2: the distance at the BCS phase $(P=0)$ is considerably larger than at any other $P>0$ (some examples shown in Fig. 2), including the FP phase $(P=1)$. That means that the FP and the normal phase (the reference) are physically closer than the BCS and the normal phases. This is not only reasonable-that magnetic phases (normal and FP) are closer than magnetic and superfluid ones-but it is also consistent with previous works [27, $43,44]$, which suggest that the BCS-normal transition is a first-order phase transition, while the normal-FP is a second-order phase transition. Thus, our distance properly reveals all the features of the system without FFLO: greater distance at $P=0$, consistent with a first-order transition, for $0.1 \lesssim P \lesssim 0.9$ an almost symmetric behavior with respect to $P=0.5$, confirming the absence of any other transition and, finally, the suggestion that from the normal to the FP, the transition is possibly of second order or simply a crossover.

Now, we consider systems with FFLO, which present, in addition to the BCS and the FP phases, the exotic superfluid phase for $0<P \leq P_{C}$ and the normal magnetic phase for $P_{C}<P<1$. The intermediate and the bottom panels of Fig. 1 present the results for the density distance in these systems. In contrast to the previous non-FFLO cases, we now see that the asymmetry appears not only at $P=0$, but also for $P>0$. This proves that our distance measure is sensing the FFLO-normal phase transition.

Figure 2 shows that while for $P>P_{C}, P_{C} \approx 0.3$, the distances behave similarly for systems with (bottom panel) or without (upper panel) FFLO, for $P<P_{C}$, the distances present distinct behaviors. Quantitatively, for $n \gtrsim 0.4$, BCS phase leads to the largest distances, followed by the FFLO-related distances and then by normal and FP-related distances. For $n \gtrsim 0.4$, in the presence of FFLO and $P<$ $P_{C}$, density distances get closer to the distances for $P=0$ and further from the ones corresponding to $P=1$. This suggests similarities between the FFLO-to-normal phase transition and the BCS-to-normal one, and would support the FFLO-to-normal phase transition being first order, like the BCS one. For smaller densities, $n \lesssim 0.4$, the increase in Coulomb interactions leads to a crossover between the $P=1$ (FP) and $P=0$ (BCS) distances (compare upper and lower panels of Fig. 2). However, FFLO-related distances remain closer to the $P=0$ curve, while normal
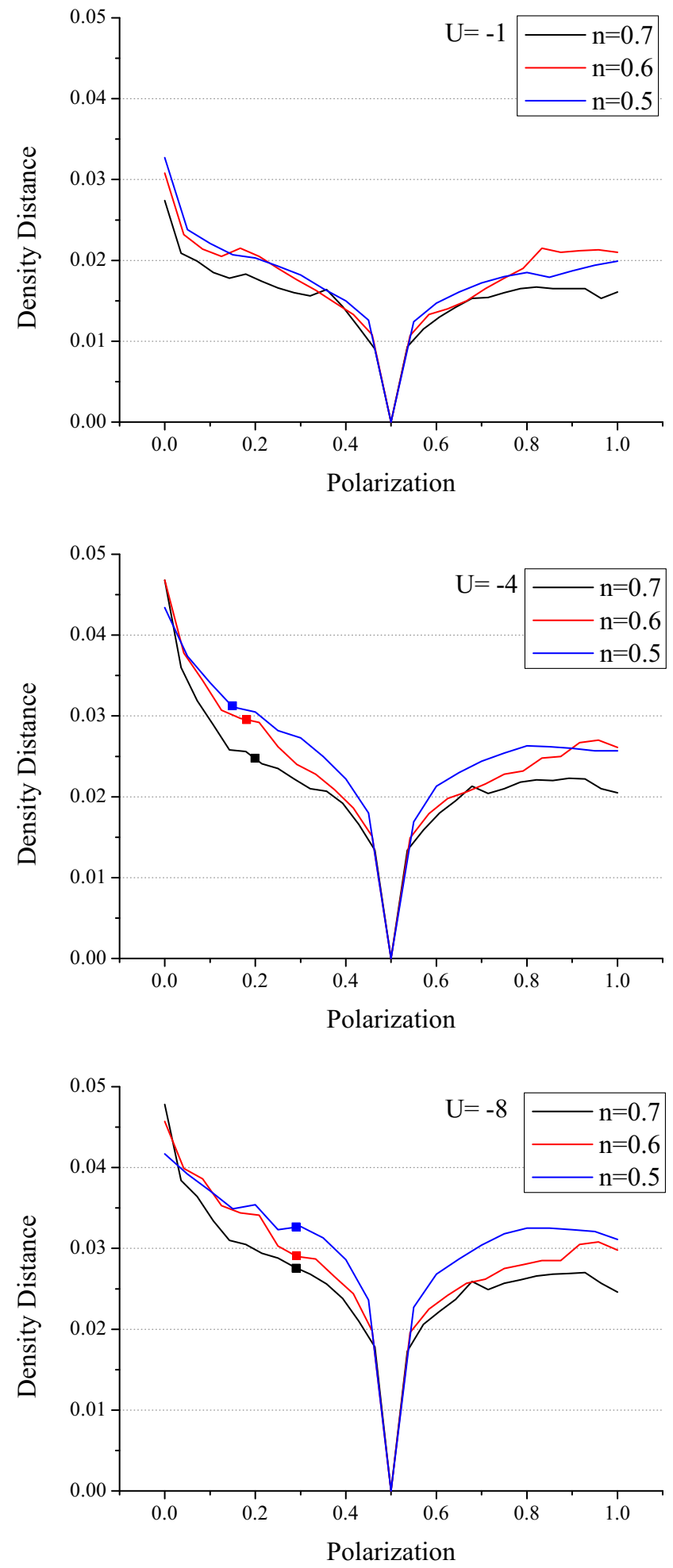

Fig. 1 Density distances $D$ as a function of $P$ for several average densities: upper panel shows non-FFLO systems, while middle and bottom panels present FFLO systems, where solid squares indicate the related $P_{C}$

phase-related distances remain closer to the $P=1$, confirming the resemblances between BCS and FFLO phases and between normal and fully polarized phases. 

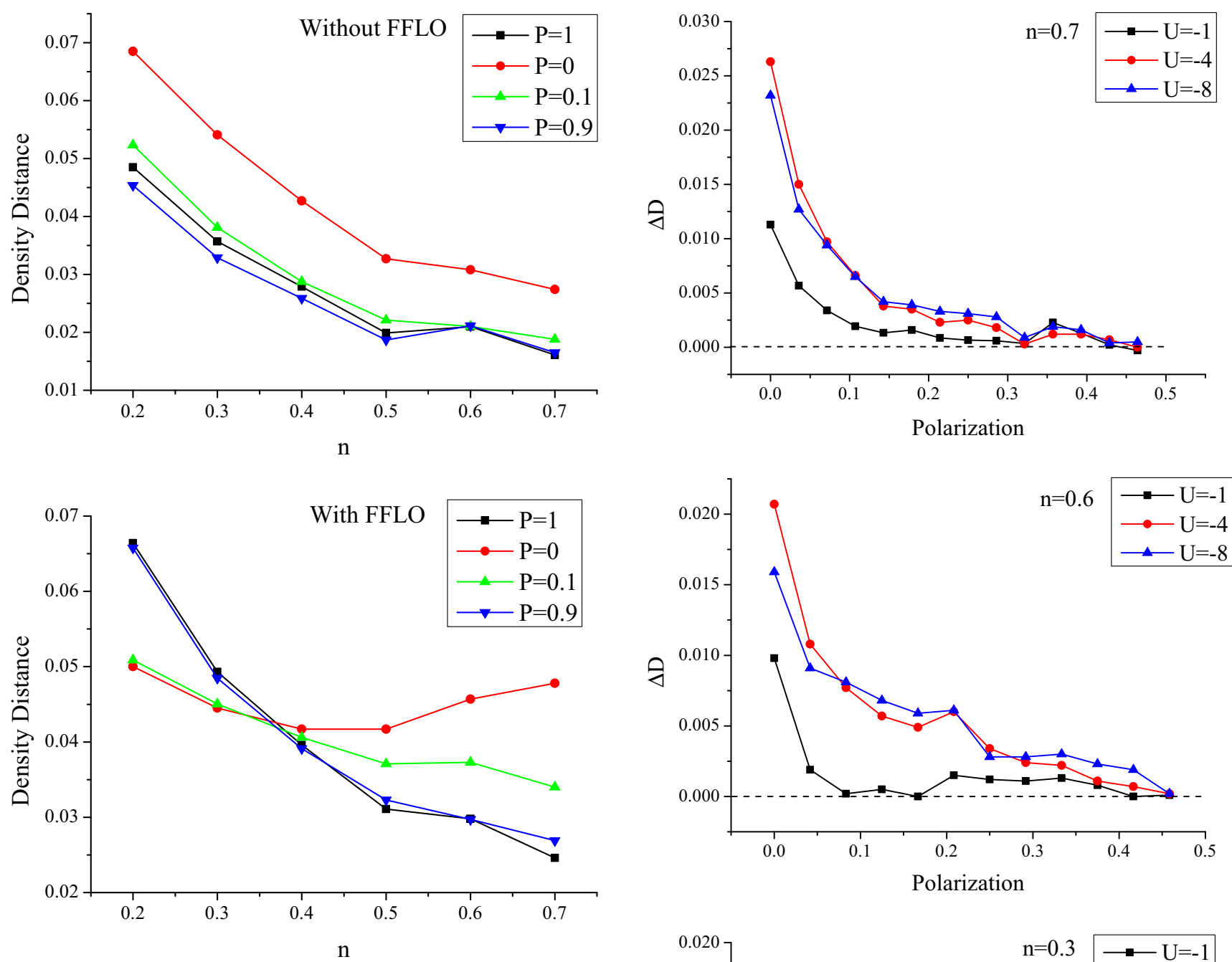

Fig. 2 Density distances as a function of the average density (filling factor). Upper panel: BCS phase $(P=0)$, normal phase $(0<P<1)$, and FP phase $(P=1)$ for $U=-1$ (without FFLO). Bottom panel: BCS phase $(P=0)$, FFLO phase $\left(0<P<P_{C}\right)$, normal phase $\left(P_{C}<P<1\right)$, and FP phase $(P=1)$ for $U=-8\left(P_{C} \sim 0.3\right.$, with FFLO)

Therefore, our distance reveals all the transitions of the system with or without FFLO: we find a distinct behavior at $P=0$ (e.g., larger distances for low $U$ and all $n$ values, see upper panel of Fig. 2) which is mimicked for $P<$ $P_{C}$, suggesting that both BCS-normal and FFLO-normal phase transitions are of first order, while we find no special behavior at $P=1$, suggesting that the normal-FP transition is a crossover or of second order.

Aiming at a quantitative comparison between the asymmetries found in Fig. 1, we explore the following difference between distances:

$\Delta D(P)=|D(1-P)-D(P)|$.

As it quantifies the asymmetry between two equidistant polarizations with respect to the central, $P=0.5$, this gives

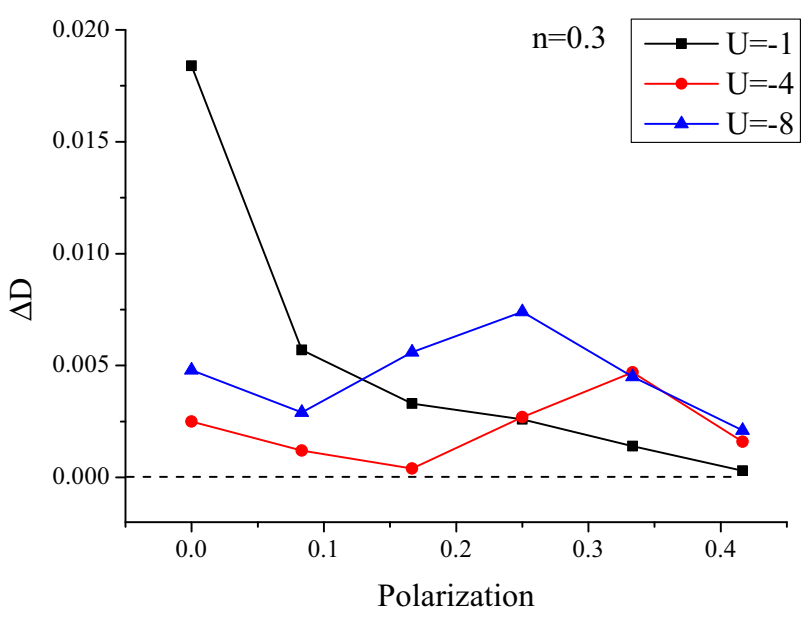

Fig. 3 Difference between equidistant density distances $\Delta D$, with respect to the central $P=0.5$, as a function of $P$ for non-FFLO systems $(U=-1)$ and FFLO systems $(U=-4$ and $U=-8)$, for distinct average densities: $n=0.7$ (upper panel), $n=0.6$ (middle panel), and $n=0.3$ (lower panel)

us a precise comparison between asymmetries for each $P$, in particular for the FFLO regime. As one can see in Fig. 3, in general, $\Delta D$ is larger for the systems with FFLO $(U=-4$ and 
$U=-8)$ than for systems without FFLO $(U=-1)$. Despite the fact that there are some fluctuations, it is clear that for non-FFLO systems (black curves), $\Delta D$ is significant only at the BCS-normal transition, while for FFLO systems (red and blue curves), it is significant also for $P>0$. For $n=0.3$ and intermediate-to-large $U$, we are close to the crossover between BCS and FP-related distances shown in the lower panel of Fig. 2), so that $\Delta D$ approaches zero. We note that $\Delta D$ is not able to identify precisely $P_{C}$, where the FFLO-normal phase transition appears. We attribute the difficulty to precisely determine $P_{C}$ to the intrinsic density fluctuations of the inhomogeneous superfluid FFLO phase: this contributes additional fluctuations to the distances and therefore a fair comparison between densities within the FFLO regime becomes impracticable.

\section{Conclusions}

In summary, we used metric-spaces analysis to probe quantum phase transitions. We find that our distances are able to distinguish among the quantum phases present in spin-imbalanced fermionic systems. Our results-via a distance measure defined with respect to a reference at the normal phase regime-suggest that both the BCSnormal and the FFLO-normal phase transitions are of first order, while the normal-FP phase transition is a smoother transition, of second order or simply a crossover. Future work includes refining the metrics such that one can identify precisely the critical $P$ values and to apply the metric-spaces approach for investigating other quantum phase transitions.

Acknowledgments VVF is financially supported by FAPESP (Grant: 2013/15982-3) and CNPq (Grant: 448220/2014-8). IDA acknowledges support from CNPq (Grant: PVE - Processo: 401414/2014-0) and from the Royal Society through the Newton Advanced Fellowship scheme (Grant no. NA140436). TP acknowledges fruitful discussions with Tatiana Pauletti. This research was supported by resources supplied by the Center for Scientific Computing (NCC/GridUNESP) of the São Paulo State University (UNESP).

\section{References}

1. S. Sachdev, Quantum phase transitions (Cambridge University Press, Cambridge, 2011)

2. A. Osterloh, L. Amico, G. Falci, R. Fazio, Nature 416, 608 (2002)

3. L. Amico, R. Fazio, A. Osterloh, V. Vedral, Rev. Mod. Phys. 80, 517 (2008)

4. V.V. França, Physica A 475, 82 (2017)
5. E. Canovi, E. Ercolessi, P. Naldesi, L. Taddia, D. Vodola, Phys. Rev. B 89, 104303 (2014)

6. J. Stasinska, B. Rogers, M. Paternostro, G. De Chiara, A. Sanpera, Phys. Rev. A 032330, 89 (2014)

7. L.-A. Wu, M.S. Sarandy, D.A. Lidar, L.J. Sham, Phys. Rev. A 74, 052335 (2006)

8. U. Marzolino, T. Prosen, Phys. Rev. B 96, 104402 (2017)

9. I. D’Amico, J.P. Coe, V.V. França, K. Capelle, Phys. Rev. Lett. 106, 050401 (2011)

10. I. D’Amico, J.P. Coe, V.V. França, K. Capelle, Phys. Rev. Lett. 107, 188902 (2011)

11. P.M. Sharp, I. D’Amico, Phys. Rev. B 89, 115137 (2014)

12. V.V. França, J.P. Coe, I. D’Amico, Sci. Rep. 8, 664 (2018)

13. P.M. Sharp, I. D'Amico, Phys. Rev. A 92, 032509 (2015)

14. P.M. Sharp, I. D’Amico, Phys. Rev. A 94, 062509 (2016)

15. I. Nagy, I. Aldazabal, Phys. Rev. 84, 032516 (2011)

16. J.P. Coe, V.V. França, I. D’Amico, EPL 110, 63001 (2015)

17. Á. Nagy, E. Romera, Rev. Phys. 88, 042515 (2013)

18. A.H. Skelt, R.W. Godby, I. D’Amico, Braz. J. Phys. (2018). https: //doi.org/10.1007/s13538-018-0589-1

19. S. Marocchi, S. Pittalis, I. D’Amico, Phys. Rev. Materials 1, 043801 (2017)

20. J. Bardeen, L.N. Cooper, J.R. Schrieffer, Phys. Rev. 106, 162 (1957)

21. P.F. Bedaque, H. Caldas, G. Rupak, Vol. 91 (2003)

22. H. Caldas, Phys. Rev. A 69, 063602 (2004)

23. P. Fulde, R.A. Ferrell, Phys. Rev. 135, A550 (1964)

24. A.L. Larkin, Y.N. Ovchinnikov, Sov. Phys. JETP 20, 762 (1965)

25. H. Mayaffre, S. Krämer, M. Horvatić, C. Berthier, K. Miyagawa, K. Kanoda, V.F. Mitrović, Nat. Phys. 10, 928 (2014)

26. Y. Liao, A.S.C. Rittner, T. Paprotta, W. Li, G.B. Partridge, R.G. Hulet, S.K. Baur, E.J. Mueller, Nature 467, 567 (2010)

27. R. Casalbuoni, G. Nardulli, Rev. Mod. Phys. 76, 263 (2004)

28. J.J. Kinnunen, J. Baarsma, J.-P. Martikainen, P. Torma, Rep. Prog. Phys. in press (2018)

29. V.V. França, D. Hörndlein, A. Buchleitner, Phys. Rev. A 033622, $86(2012)$

30. D.-H. Kim, J.J. Kinnunen, J.-P. Martikainen, P. Törmä, Phys. Rev. Lett. 106, 095301 (2011)

31. G.G. Batrouni, M.H. Huntley, V.G. Rousseau, R. Scalettar, Phys. Rev. Lett. 100, 116405 (2008)

32. H. Caldas, M.A. Continentino, J. Phys. B: At Mol. Opt. Phys. 46, $155301(2013)$

33. J. Hubbard, Proc. R. Soc. London. Series A 276, 238 (1963)

34. K. Capelle, L.C. Jr.. Vivaldo, Phys. Rep. 528, 91 (2013)

35. J. Fernández-Rossier, J.J. Palacios, Phys. Rev. Lett. 99, 177204 (2007)

36. J.P. Coe, V.V. França, I. D’Amico, Phys. Rev. A 81, 052321 (2010)

37. O.V. Yazyev, Rep. Prog. Phys. 73, 056501 (2010)

38. J.P. Coe, V.V. França, I. D'Amico, EPL 93, 10001 (2011)

39. J.P. Coe, V.V. França, I. D'Amico, J. Phys. Conf. Series 286, 012048 (2011)

40. T. Mendes-Santos, T. Paiva, R.R. dos Santos, Phys. Rev. B 87, 214407 (2013)

41. V.V. França, I. D’ Amico, Phys. Rev. A 83, 042311 (2011)

42. U. Schollwöck, Rev. Mod. Phys. 77, 259 (2005)

43. S. Pilati, S. Giorgini, Phys. Rev. Lett. 100, 030401 (2008)

44. D.E. Sheehy, L. Radzihovsky, Ann. Phys. 322, 1790 (2007) 